\title{
Phlebotominae (Diptera: Psychodidae) de Lagoas, município de Buriticupu, Amazônia Maranhense. I - Riqueza e abundância relativa das espécies em área de colonização recente
}

\author{
Sand flies (Diptera: Psychodidae) of Lagoas, municipal district of Buriticupu, \\ Amazonia of Maranhão. I - Richness and relative abundance of the \\ species in area of recent colonisation
}

\begin{abstract}
José Manuel Macário Rebêlo', Sueli Trindade de Oliveira33, Vera Lúcia Lopes Barros ${ }^{3}$, Francinaldo Soares Silva ${ }^{3}$, Jackson Maurício Lopes Costa ${ }^{2}$, Luiz Alves Ferreira ${ }^{2}$ e Antonio Rafael da Silva ${ }^{2}$
\end{abstract}

\begin{abstract}
Resumo Este estudo determinou a riqueza de espécies e a abundância relativa dos flebotomíneos da comunidade de Lagoas, em Buriticupu, na Amazônia Maranhense. O estudo consistiu na captura de formas adultas com auxílio de armadilhas luminosas tipo CDC e armadilha de Shannon, das 18 às 6 horas, uma vez por mês, de dezembro/95 a janeiro/97, em ambiente florestal e extraflorestal (peri e intradomicílios). No total, capturou-se 9.392 espécimens (4.302 machos e 5.090 fêmeas) distribuídos em 38 espécies (1 Brumptomyia e 37 Lutzomyia). A riqueza e abundância de espécies foram maiores na mata, seguida do peri e intradomicílio. Na mata, as espécies mais freqüentes foram Lutzomyia whitmani (64,9\%), L. migonei (27\%) e L. serrana $(3,6 \%)$. No peridomicílio, predominaram L. evandroi $(55,4 \%)$, L. whitmani $(33,4 \%)$ e L. migonei $(6,4 \%)$ e no intra, L. evandroi (97,9\%). O predomínio no ambiente silvestre resulta das potencialidades ainda encontradas nas glebas remanescentes da floresta tão explorada pelas atividades madeireira e agropecuária. Por outro lado, a peridomiciliação de $7,7 \%$ das espécies reverteu-se de grande importância epidemiológica, haja visto a existência de pacientes com leishmanioses que julgam ter adquirido a infecção nas proximidades de suas habitações.
\end{abstract}

Palavras-chaves: Phlebotominae. Lutzomyia. Leishmaniose. Amazônia Maranhense

\begin{abstract}
In this study the diversity of the species and relative abundance of the sand flies in the Amazonian community of Lagoas, in Buriticupu, Maranhão State, was determined. The study consisted of adult sandflies captured with CD light-traps, Shannon-trap and manual aspiration tube, between 18 PM and 6 AM, once a month, from December/95 to January/97, in a forest fragment and in the domestic environment (peri and intra). In all, 9,393 specimens were captured (4,302 males and 5,140 females) distributed among 38 species (1 Brumptomyia and 37 Lutzomyia). The diversity and abundance of species was higher in the forest, followed by the peri and intra. The predominance in the wild ambient is a consequence of the potentialities found in the remaining fragments of the forest which have survived the lumber yard exploitation and agricultural activities. In the forest environment Lutzomyia whitmani (64.9\%), L. migonei (27\%) and L. serrana (3.6\%) were the most frequent species. In the peri and intra domicile $\mathrm{L}$. evandroi predominated $(55.4 \%$ and $97.9 \%)$. The species, L. whitmani $(33.4 \%)$ and L. migonei (6.4\%), were important in the peri domicile. The peridomestic habit of $7.7 \%$ of the sand flies showed the greatest epidemiologic importance, due to the existence of patients with leishmaniasis ulcers, who believe they were infected near their habitations.
\end{abstract}

Key-words: Phlebotominae. Lutzomyia. Leishmaniasis. Amazonia of Maranhão.

\footnotetext{
1. Departamento de Biologia, da Universidade Federal do Maranhão, São Luís, MA. 2. Departamento de Patologia da Universidade Federal do Maranhão, São Luís, MA. 3. Laboratório de Entomologia e Vetores da Universidade Federal do Maranhão, São Luís, MA. Endereço para correspondência: Dr. José Manuel Macário Rebêlo. Departamento de Patologia/UFMA. Núcleo de Patologia Tropical e Medicina Social. Praça Madre Deus 2. 65025-560 São Luís, MA, Brasil

Fax: 5598 232-3837.

Recebido para publicação em 6/10/98.
} 
A leishmaniose tegumentar americana (LTA) representa um dos grandes problemas de saúde pública no Brasil. Para se ter uma idéia, basta verificar que de 1980 até 1993 foram notificados mais de 153 mil casos oriundos de todas as unidades do Brasil, exceto do Distrito Federal ${ }^{18}$. $\mathrm{Na}$ Amazônia, uma das regiões mais atingidas do Brasil, a LTA tem sido descrita como doença zoonótica ${ }^{12}$, sendo a população mais acometida representada pelos lavradores em atividade de desmatamento ou extração de madeiras, pelos coletores de látex e outros produtos naturais da floresta e por aqueles que trabalham na construção de novas estradas ${ }^{11}$.

$\mathrm{Na}$ Amazônia Maranhense, há muito se conhece a existência de áreas endêmicas de leishmanioses. Em 1979, Silva et al ${ }^{24}$ estudaram um surto com 300 casos de LTA, ocorridos em área de colonização recente para implantação de projetos agropastoris, em Buriticupu. Desde então, essa região, caracterizada pela presença de densas matas amazônicas, vem sofrendo intenso processo de desflorestamento, acompanhado com o aumento da notificação de casos de LTA ${ }^{5}$.

A existência desta enfermidade em Buriticupu vem causando sérias preocupações, pois além de se constituir em uma das principais endemias da região ${ }^{4}$, se desconhece os vetores e as circunstâncias com que vem ocorrendo o processo de transmissão.

Até o momento, não existe nenhum trabalho sobre a composição da fauna dos flebotomíneos vetores e sua dinâmica nesta região do Estado. Aliás, o único trabalho conhecido abordando o estudo da composição faunística destes insetos no Maranhão foi realizado por Rebêlo et al ${ }^{22}$.

Alguns trabalhos sobre a composição da fauna e a ecologia de flebotomíneos em ambientes florestal e extraflorestal (peri e intradomicílios) estão em andamento, de forma que, no futuro próximo ter-se-á uma estimativa razoável sobre a fauna flebotomínica de Buriticupu.

Neste trabalho, apresenta-se a riqueza e a abundância relativa de espécies de flebotomíneos presentes na localidade de Lagoas, área de colonização recente da região de Buriticupu. Pretende-se contribuir para ampliar os conhecimentos sobre estes importantes vetores e indicar meios que possam ajudar os órgãos de saúde e as comunidades no sentido de minimizar o contato homem-vetor e, desta forma, controlar o avanço da leishmaniose.

\section{MATERIAL E MÉTODOS}

Área de Estudo. O estudo foi realizado no município de Buriticupu situado entre $4^{\circ}$ e $5^{\circ}$ LS e $45^{\circ} 30^{\prime}$ e $47^{\circ} \mathrm{LW}$, na Amazônia Maranhense (Figura 1) a uma altitude média de 200 metros.

O relevo da região de Buriticupu é derivado da Formação Itapecuru (do Cretáceo Inferior), que se estende praticamente por toda a metade norte do Estado, ocupando uma área de cerca de $50 \%$ do território estadual. Esta formação é constituída por arenitos finos, avermelhados, róseos, cinza-argilosos, com abundante silicificação na parte superior. Em alguns pontos, apresenta afloramento de tabuleiros isolados do Grupo Barreira ${ }^{10}$.

O solo é o latossolo amarelo, ocorrendo tanto na área dos tabuleiros terciários, quanto nos platôs cretácicos. A textura varia em função da situação topográfica e do material de origem. Pode ser constituída por sedimentos argilosos, argilo-arenosos ou arenosos. São solos velhos, ácidos, com boa drenagem e permeáveis ${ }^{10}$.

A cobertura vegetal original da região de Buriticupu, bastante devastada pela atividade madeireira e projetos agropecuários, é constituída por floresta. Toda a região noroeste do Maranhão é recoberta pela floresta que corresponde a região de Tocantins e do Gurupi, descrita por Hueck $^{9}$ e se prolonga do rio Gurupi ao Mearim, passando pelo Pindaré. Pode-se distinguir dois tipos florestais nesta região do Estado: a densa, que corresponde à floresta de dossel fechado e a aberta, que corresponde à chamada mata de cipó ou cipoal, em grande parte envolvida por lianas, com árvores também de menor altura. Em certos trechos, principalmente no vale do Gurupi, a floresta atinge sua forma mais exuberante no Maranhão. Para leste ela vai se tornando menos exuberante e com tendência a uma perda substancial de folhas no período mais seco. Nestas áreas surge a presença, em maior ou menor número, de palmeiras babaçu. A floresta da região de Buriticupu fica numa situação intermediária, sendo constituída de árvores altas e grossas, que podem atingir até 50 metros ou mais, característica de floresta ombrófila dos platôs, ocupando áreas sedimentares altas e baixas. Atualmente, os remanescentes da 


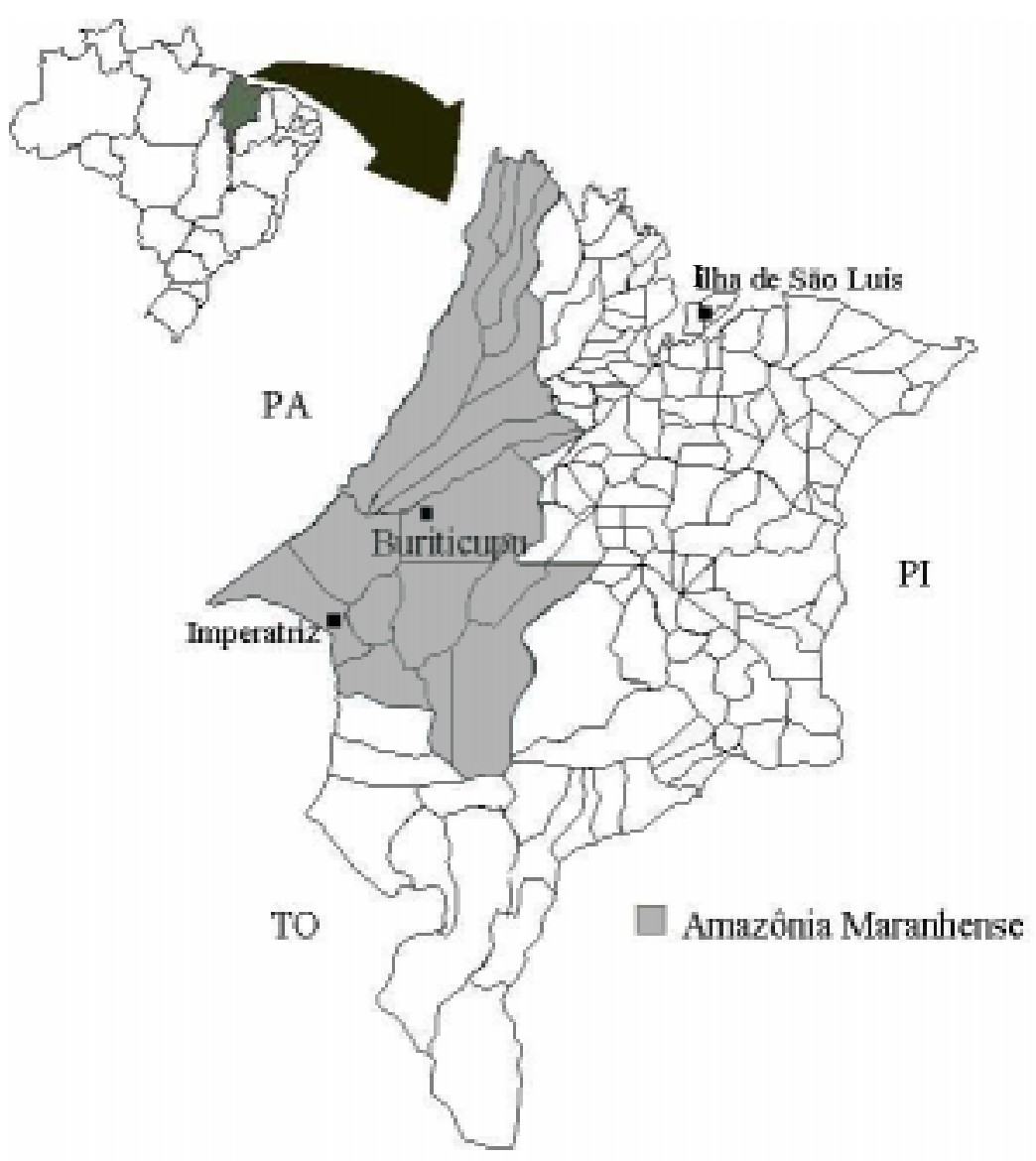

Figura 1 - Localização de Buriticupu na Amazônia Maranhense.

vegetação original estão representados por glebas de matas isoladas sobre alguns platôs. Antigamente não havia extratos arbustivos, sendo as plantas de porte menor da submata, árvores jovens, resultantes de matrizes próximas. Entretanto, o que se presencia hoje em várias áreas é o domínio de capoeiras e de espécies arbustivas nas matas depredadas.

O rio Pindaré, o principal curso d'água da região e um dos formadores da bacia do Mearim, possui aproximadamente $468 \mathrm{~km}^{10}$. As suas margens, outrora cobertas por matas ciliares, sofreram processo intenso de desmatamento nos últimos anos, contribuindo para a elevação do índice de evaporação especialmente no período de estiagem.

A região de Buriticupu está subordinada ao clima quente-úmido de transição entre o super- úmido amazônico e o semi-árido nordestino, com 6 meses secos e precipitação média anual em torno de $1800 \mathrm{~mm}^{17}$.

O estudo foi realizado em uma área florestal, na qual encontra-se assentada há 12 anos, a comunidade de Lagoas. As capturas foram realizadas na floresta e nos ambientes intra e peridomiciliares (galinheiro e chiqueiro). O local da mata, selecionado para o estudo, com aproximadamente $6 \mathrm{ha}$, distava cerca de $7 \mathrm{~km}$ do povoado de Lagoas e $60 \mathrm{~km}$ da sede municipal de Buriticupu.

Método de coleta. Os flebotomíneos foram capturados das 18 às 6 horas, mensalmente, de dezembro/95 a janeiro/97, na floresta, no peri e intradomicílios. No ambiente florestal utilizou-se uma armadilha luminosa tipo CDC e optou-se, também, pela realização de capturas manuais 
utilizando-se da armadilha de Shannon. No ambiente peridomiciliar foram utilizadas somente armadilhas CDC, uma no galinheiro e a outra no chiqueiro. No domicílio utilizou-se apenas uma armadilha CDC no dormitório. As armadilhas eram colocadas a uma altura de $1,5 \mathrm{~m}$ do nível do solo.

Todos os exemplares capturados eram separados por horário, mortos em câmaras de acetato de etila e acondicionados em frascos contendo álcool a $70 \%$ para posterior transporte ao laboratório, onde eram montados e identificados.

Utilizou-se teste estatístico não-paramétrico (qui-quadrado) para analisar as diferenças entre os flebotomíneos capturados nos diferentes ambientes. As diferenças foram consideradas significativas quando a probabilidade $(p)$ do erro foi inferior a $1 \%(p<0,01)$.

\section{RESULTADOS}

Riqueza de espécies. Encontrou-se 38 espécies de flebotomíneos, sendo uma do gênero Brumptomyia França \& Parrot, 1921 e 37 do gênero Lutzomyia França, 1924 (Tabela 1).

Tabela 1 - Flebotomíneos encontrados no ambiente florestal e extraflorestal (intra e peridomicílios), na localidade de Lagoas, zona rural de Buriticupu, Amazônia Maranhense, de dezembro/95 a janeiro/97.

\begin{tabular}{|c|c|c|c|c|c|c|c|}
\hline \multirow[t]{2}{*}{ Flebotomíneos } & \multicolumn{2}{|c|}{ Mata } & \multicolumn{2}{|c|}{ Peri } & \multicolumn{2}{|c|}{ Intra } & \multirow{2}{*}{$\begin{array}{r}\text { Total } \\
\text { Ind. }\end{array}$} \\
\hline & $M$ & $\mathrm{~F}$ & $M$ & $\mathrm{~F}$ & $M$ & $\mathrm{~F}$ & \\
\hline L. whitmani & 2316 & 3066 & 179 & 62 & 1 & - & 5624 \\
\hline L. migonei & 1065 & 1181 & 17 & 29 & 3 & 1 & 2296 \\
\hline L. evandroi & 15 & 14 & 216 & 184 & 257 & 113 & 799 \\
\hline L. serrana & 9 & 294 & - & 1 & - & 1 & 305 \\
\hline L. umbratilis & 50 & 57 & - & - & - & - & 107 \\
\hline L. shannoni & 63 & 17 & - & 1 & - & - & 81 \\
\hline L. claustrei & 66 & - & 1 & 2 & - & - & 69 \\
\hline L. termitophila & - & 7 & 3 & 16 & - & 1 & 27 \\
\hline L. furcata & 2 & 10 & - & 2 & - & - & 14 \\
\hline L. dendrophyla & 11 & 1 & - & - & - & - & 12 \\
\hline L. hirsuta hirsuta & 6 & - & - & - & - & - & 6 \\
\hline L. longipalpis & 4 & - & 2 & - & - & - & 6 \\
\hline B. avellari & 5 & 1 & - & - & - & - & 6 \\
\hline L. brachyphalla & 3 & 1 & - & - & - & - & 4 \\
\hline L. choti & - & 1 & - & 3 & - & - & 4 \\
\hline L. geniculata & - & 3 & - & - & - & - & 3 \\
\hline L. goiana & 1 & 1 & - & - & - & - & 2 \\
\hline L. gomezi & 1 & - & - & 1 & - & - & 2 \\
\hline L. corossoniensis & - & 2 & - & - & - & - & 2 \\
\hline L. punctigeniculata & - & 1 & - & 1 & - & - & 2 \\
\hline L. saulensis & - & 2 & - & - & - & - & 2 \\
\hline L. spathotrichia & - & 2 & - & - & - & - & 2 \\
\hline L. trinidadensis & 2 & - & - & - & - & - & 2 \\
\hline L. abonnenci & - & 1 & - & - & - & - & 1 \\
\hline L. arthuri & - & 1 & - & - & - & - & 1 \\
\hline L. ayrozai & - & 1 & - & - & - & - & 1 \\
\hline L. brasiliensis & - & 1 & - & - & - & - & 1 \\
\hline L. carrerai carrerai & - & 1 & - & - & - & - & 1 \\
\hline L. complexa & - & 1 & - & - & - & - & 1 \\
\hline L. aff. falcata & 1 & - & - & - & - & - & 1 \\
\hline L. aff. gomezi & - & - & - & - & 1 & - & 1 \\
\hline L. nevesi & - & - & - & 1 & - & - & 1 \\
\hline L. sordellii & - & 1 & - & - & - & - & 1 \\
\hline L. paraensis & - & 1 & - & - & - & - & 1 \\
\hline L. (Psychodopygus) sp. & 1 & - & - & - & - & - & 1 \\
\hline L. scaffi & - & 1 & - & - & - & - & 1 \\
\hline L. servulolimai & - & 1 & - & - & - & - & 1 \\
\hline L. trispinosa & - & - & 1 & - & - & - & 1 \\
\hline № indivíduos & 3622 & 4671 & 419 & 303 & 261 & 116 & 9392 \\
\hline № espécies & & & & & & & \\
\hline
\end{tabular}

$\mathrm{M}=$ machos $\mathrm{F}=$ fêmeas 
O predomínio foi do subgênero Psychodopygus Mangabeira, 1941, representado por 10 espécies, seguido pelos subgêneros Evandromyia Mangabeira, 1941b, Lutzomyia França, 1924 e grupo Migonei, com 4 espécies cada um. O subgênero Psathyromyia Barreto, 1962 e o grupo Brasiliensis foram representados por 3 espécies; Nyssomyia Barreto, 1962, Pressatia Mangabeira, 1942 e os grupos Oswaldoi e Verrucarum tiveram duas espécies cada um e o grupo Saulensis uma espécie.

Abundância relativa. Foram capturados 9.392 espécimens, sendo 5.090 fêmeas e 4.302 machos (Tabela 1). A análise dos números totais de indivíduos revelou diferenças significativas $(p$ $<0,01$ ) na abundância entre os sexos, sendo as fêmeas significativamente mais abundantes (Figura 2A).

A espécie mais abundante foi $L$. whitmani, sendo representada por $59 \%$ dos espécimens capturados (5.624 espécimens: 2.496 machos e 3.128 fêmeas). Seguem na ordem de importância L. migonei (24,5\%, 2.296: 1085 e 1211), L. evandroi (8,5\%, 799: 488 e 311), L. serrana (3,3\%, 305: 9 e 296) e L. umbratilis (1,1\%, 107:50 e 57). As demais espécies representaram juntas 3,6\% da amostra total (Figura 2B).
Ambiente florestal. Na mata foram encontradas 35 espécies e 8.293 espécimens, sendo 3.622 machos e 4.671 fêmeas, resultando em $88,3 \%$ dos espécimens capturados (Figura 2C). A análise dos números totais de machos e fêmeas capturados neste ambiente não revelou diferenças significativas na abundância entre os sexos. As espécies dominantes foram L. whitmani, representando $64,9 \%$ dos exemplares capturados neste ambiente (2.316 machos e 3.066 fêmeas), L. migonei com 27\% (1.065 machos e 1.181 fêmeas), L. umbratilis com 1,3\% (50 machos e 57 fêmeas), L. shannonicom 1,0\% (63 machos e 17 fêmeas) e L. claustreicom 0,8\% (66 machos). As demais espécies representaram juntas $5 \%$ da amostra (Figura $3 \mathrm{~A}$ ). Entretanto, L. whitmani foi significativamente mais abundante do que todas as outras espécies juntas. Ressaltase que L. claustrei foi a única espécie representada exclusivamente por machos. As demais por ambos os sexos, sendo que o número de machos capturados foi significativamente maior em L. whitmani e L. shannoni, não havendo diferenças significativas entre os sexos para L. migonei e L. umbratilis.

Ambiente peridomiciliar. No peridomicílio foram encontradas 14 espécies e 722 espécimens,

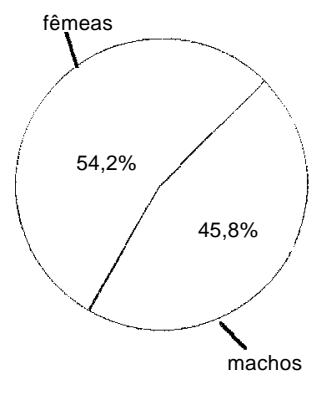

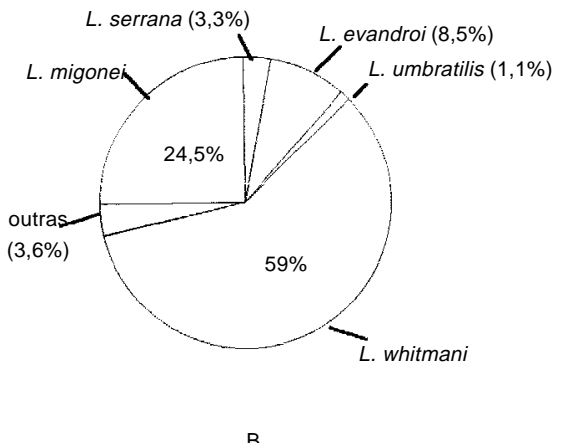

B

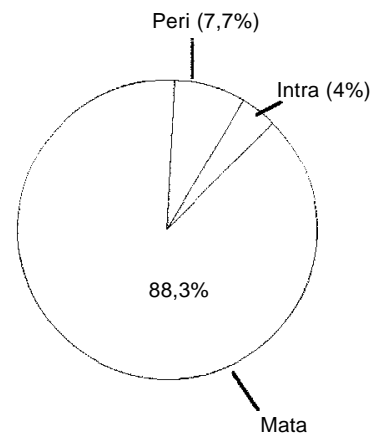

Figura 2 - Números percentuais de flebotomíneos capturados na localidade de Lagoas, município de Buriticupu, Amazônia Maranhense, de dezembro/95 a janeiro/97.

sendo 419 machos e 303 fêmeas, totalizando $7,7 \%$ dos espécimens capturados no estudo (Figura 2C). O número global de machos capturados foi significativamente maior. As espécies mais freqüentes foram $L$. evandroicom $55 \%$ dos exemplares capturados neste ambiente, sendo 216 machos e 184 fêmeas; L. whitmani com 33,4\%, 179 machos e 62 fêmeas e $L$. migonei com 6,4\% (17 machos e 29 fêmeas). As demais espécies representaram juntas 5,2\% (Figura 3B). Somente L. whitmani apresentou o número de machos significativamente maior do que o de fêmeas, não havendo diferenças significativas para $L$. evandroi e $L$. migonei. 
Ambiente intradomiciliar. No intradomicílio foram encontradas 5 espécies e 427 espécimens, sendo 261 machos e 166 fêmeas, totalizando $4 \%$ dos espécimens capturados (Figura 2C). A espécie mais freqüente foi $L$. evandroi, englobando $86,7 \%$ dos exemplares capturados (257 machos e 113 fêmeas). As demais espécies representaram juntas $13,3 \%$ da amostra (Figura $3 \mathrm{C}$ ).
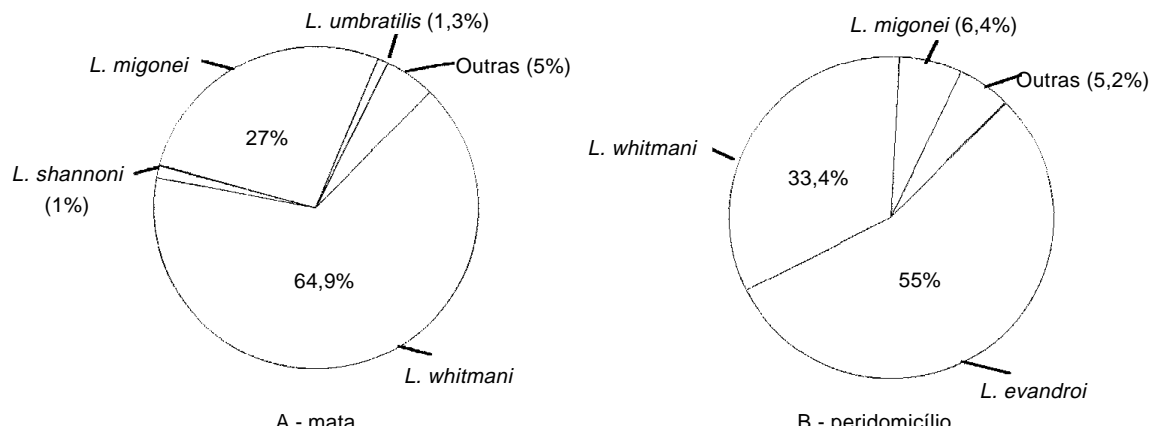

A - mata

B - peridomicílio

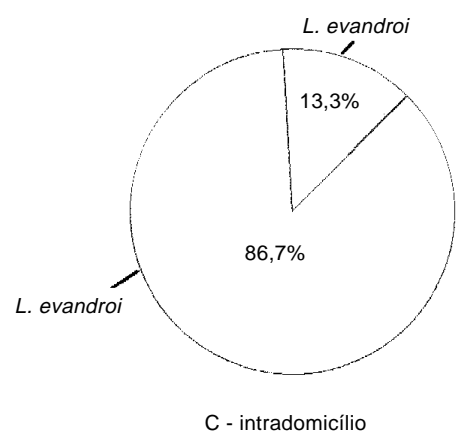

Figura 3 - Números percentuais de flebotomíneos capturados de acordo com o ambiente, na localidade de Lagoas, município de Buriticupu, Amazônia Maranhense, de dezembro/95 a janeiro/97.

\section{DISCUSSÃO}

A fauna de flebotomíneos encontrada em Lagoas mostrou-se bastante diversificada, constituída de um grande número de espécies, pertencentes a diferentes grupos e com padrões distintos de distribuição. A julgar pelas características amazônicas da área de estudo, não foi surpresa constatar a existência de uma alta similaridade entre a fauna de Lagoas e aquela encontrada nas áreas florestais do vizinho Estado do Pará ${ }^{3}$. O índice de similaridade observado foi em torno de $89,2 \%$, ou seja, 33 espécies presentes em Lagoas têm registros prévios de ocorrência no Pará. Apesar desta grande similaridade entre as faunas amazônicas maranhense e paraense, poucas espécies podem ser consideradas tipicamente amazônicas.
Muitas, no entanto, distribuem-se também em áreas não amazônicas ${ }^{14}$, enquanto outras, ao que consta, não têm registros na Amazônia Paraense ${ }^{23}$, são próprias de áreas mais secas, do Nordeste ou do Centro-Sul do Brasil.

Das espécies encontradas em Lagoas com distribuição restrita às áreas amazônicas podese citar $L$. complexa e $L$. umbratilis, duas espécies importantes do ponto de vista da saúde pública. A primeira tem sido incriminada como o principal vetor de Le. braziliensis, em áreas livres da ocorrência de L. wellcomei, na Amazônia Paraense ${ }^{25}$. A segunda, é reconhecidamente o vetor primário da Le. guyanensis na Bacia Amazônica. Em Lagoas, ambas foram encontradas somente no ambiente florestal e pobremente representadas. 
De acordo com as observações de vários autores $^{21320}$, é durante a noite que $L$. umbratilis prefere praticar a hematofagia e o faz nas copas das árvores, onde estão os seus hospedeiros naturais. Como neste trabalho as capturas foram realizadas ao nível do solo, talvez seja esse o motivo da baixa freqüência com que foram encontradas nas armadilhas. De qualquer forma, o registro de ambas as espécies na área já serve de alerta.

Dentre as espécies encontradas em Lagoas, com ampla distribuição no país, tem-se: $B$. avellari, L. migonei, L. serrana, L. whitmani, $L$. evandroi, L. ayrozai, L. hirsuta, L. longipalpis e $L$. shannoni, todas encontradas na Amazônia e no Centro-Sul do Brasil 3151277 . A ampla distribuição de L. whitmani e seu papel na transmissão de Le. braziliensis já é bem conhecida. A presença de L. longipalpis em Lagoas é digno de nota, pois trata-se do vetor da Le. chagasi, o agente etiológico do calazar no Brasil. A região de Buriticupu, até o momento, encontra-se livre desta forma de leishmaniose, contudo, não se pode esquecer que o calazar, por um lado, é endêmico na ilha de São Luís e nos municípios do baixo Parnaíba ${ }^{16}$, com características nordestinas, e por outro, já foi introduzido no vizinho município de Imperatriz, com características amazônicas. Por esses motivos, atenção especial deve ter a Vigilância Sanitária, pois Buriticupu é rota obrigatória para o fluxo migratório entre os municípios destas áreas.

Entre as espécies que distribuem-se nas áreas que abrangem o Nordeste e o Centro-Sul do Brasil ${ }^{14}$, e agora com registros em Lagoas, estão L. arthuri, L. goiana, L. nevesie L. termitophila.

O padrão de abundância dos flebotomíneos em Lagoas foi similar àquele que tem sido encontrado em qualquer levantamento de insetos, ou seja, com o predomínio das espécies com poucos indivíduos, sobre aquelas mais abundantes. Este padrão resultou em acentuada diferença no número de exemplares capturados entre as espécies. Também observou-se o predomínio de fêmeas sobre os machos. No ambiente silvestre várias espécies foram representadas exclusivamente por machos ou estes superaram as fêmeas em números de indivíduos. No ambiente peridomiciliar, por exemplo, L. evandroie L. whitmani tiveram mais machos do que fêmeas atraídos às armadilhas. Nestes casos, considera-se que os machos atraídos às armadilhas estavam acompanhando as fêmeas para a cópula, pois, é sabido que machos de flebotomíneos formam agregados com o propósito de acasalamento ${ }^{6}$.

Em Lagoas houve uma tendência dos flebotomíneos preferirem o ambiente florestal. Este padrão ficou claro quando se observou que das 38 espécies encontradas, $35(94,7 \%)$ ocorreram na mata, enquanto $14(36,8 \%)$ estavam no ambiente extraflorestal com 6 $(15,8 \%)$ ocorrendo dentro das habitações humanas. Observou-se também que 11 espécies estiveram presentes tanto no ambiente florestal quanto no extra florestal. Neste grupo, as espécies tenderam a apresentar maior densidade no ambiente florestal, destacando-se $L$. whitmani, L. migonei, L. serrana, L. shannonie L.claustrei; enquanto $L$. evandroi e $L$. termitophila tenderam a ocorrer com maior abundância no ambiente domiciliar.

O flebotomíneo mais comum em Lagoas, $L$. whitmani, dominou no ambiente silvestre, sendo a segunda espécie mais freqüente no ambiente peridomiciliar. Este achado é interessante, pois na Amazônia brasileira há uma tendência desta espécie ocorrer em alta densidade nas florestas, ao contrário do que ocorre no ambiente domiciliar, onde ao que consta não se tem achado exemplares ${ }^{1921}$. Por outro lado, em áreas não amazônicas como em Três Braços, Bahia ${ }^{27}$ e em Caratinga, Minas Gerais ${ }^{15}$, é a espécie mais comum nos ambientes peri e intradomicliares. Neste aspecto, a população de $L$. whitmani da região de Buriticupu parece pertencer a uma zona de transição entre as populações amazônica, típica de floresta e não amazônica peridomiciliadas. No Nordeste e no Centro-Sul do Brasil, L. whitmani adaptou-se fortemente ao ambiente peridomiciliar, certamente devido ao intenso desflorestamento que se processou ao longo do tempo, de modo que tem sido encontrada em densidade muito baixa no ecótopo natura ${ }^{27}$. Este comportamento ainda não está ocorrendo com a população de Lagoas, pois apesar de forte hábito peridomiciliar, ainda conserva uma consistente colonização silvestre. Na região Sul, juntamente com $L$. migonei têm predominado em área florestal primária, no município de Londrina- $\mathrm{PR}^{8}$ e em mata residual, no município de Terra Boa, $\mathrm{PR}^{26}$, fugindo do padrão que tem sido observado para as áreas não amazônicas do Brasil. Vários estudos realizados no Brasil têm demonstrado que $L$. whitmani pode funcionar como vetor de mais espécies de Leishmania'111213. 
L. migonei também foi muito comum no ecótopo silvestre e a espécie mais freqüente no ambiente domiciliar. Infelizmente, não se dispõe de dados sobre infecção por Leishmania, todavia, Lainson \& Shaw ${ }^{13}$ fazem referência do encontro de Le. braziliensis por Pessôa \& Pestana, em São Paulo. Devido a elevada densidade com que foi observada, passa a adquirir papel de relevância do ponto de vista epidemiológico, pois pode vir a ter importância na transmissão de Leishmania ao homem, na região de Lagoas.

$L$. evandroi também se destacou entre as espécies mais abundantes, sendo a mais comum nos ambientes peri e intradomiciliares. O número de exemplares capturados nestes dois ambientes foi maior do que a somatória dos exemplares de todas as outras espécies juntas. Levando-se em consideração o fator densidade, como um dos requisitos no processo da transmissibilidade da infecção leishmaniótica, atenção especial deve ser dada a esta espécie.
O que pode-se concluir, com este trabalho, em primeiro lugar, é que ele marca o início do estudo dos flebotomíneos da Amazônia do Maranhão. A partir das espécies encontradas em Lagoas, tem-se uma noção razoável da composição da fauna e, a julgar pela diversidade, a região de Buriticupu adquire grande importância por apresentar em seu território zonas de superposição de espécies de regiões biogeográficas distintas, fato incomum em outras áreas onde os flebotomíneos têm sido estudados. Em segundo lugar, com os resultados aqui obtidos amplia-se o conhecimento acerca da distribuição de espécies que reconhecidamente atuam como vetor das leishmanioses e os tipos de ambientes em que podem ser encontradas. A existência de várias espécies consideradas vetoras de agentes etiológicos da LTA no peridomicílio explicaria a existência de leishmaniose em indivíduos que não têm o hábito de entrar na mata.

\section{AGRADECIMENTO}

Ao Ministério da Saúde pelo auxílio concedido na realização deste trabalho.

\section{REFERÊNCIAS}

1. Aguiar GM, Schuback PA, Vilela ML, Azevedo ACR Aspectos da ecologia dos flebótomos do Parque Nacional da Serra dos Órgãos, Rio de Janeiro. II. Distribuição vertical (Diptera, Psychodidae, Phlebotominae). Memórias do Instituto Oswaldo Cruz 80:187-194, 1985.

2. Arias JR, Freitas RA. Sobre os vetores de leishmaniose cutânea na Amazônia Central do Brasil. 2. Incidência de flagelados em flebótomos selváticos. Acta Amazônica 8:387-396, 1978.

3. Arias JR, Freitas RA. On the vectors of cutaneous leishmaniasis in the Central amazon of Brazil. 3. Phlebotomine sand fly stratification in a terra firme forest. Acta Amazônica 12:599-608, 1982.

4. Costa JML, Balby ITA, Rocha JES, Silva AR, Rebêlo JMM, Ferreira LA, Gama MEA, Branco MRFC, Burattini MN, Soares NJ. Estado comparativo da leishmaniose tegumentar americana em crianças e adolescentes procedentes das áreas endêmicas de Buriticupu (Maranhão) e Corte de Pedra (Bahia), Brasil. Revista da Sociedade Brasileira de Medicina Tropical 25:115-123, 1998.

5. Costa JML, Saldanha ACR, Mello-Silva AC, Serra-Neto A, Galvão CES, Pedroso-Silva CM, Silva AR. Estado atual da leishmaniose cutânea difusa (LCD) no Estado do Maranhão. II. Aspectos epidemiológicos, clínicos e evolutivos. Revista da Sociedade Brasileira de Medicina Tropical 25:115-123, 1992.
6. Feliciangeli MD. Ecology of sandflies (Diptera: Psychodidae) in a restricted focus of cutaneuos Leishmaniasis in northern Venezuela. I. Description of the study area, catching methods and species composition. Memórias do Instituto Oswaldo Cruz 82:119-124, 1987.

7. Galati EAB, Nunes VLB, Dorval MEC, Oshiro ET, Cristaldo G, Espínola MA, Rocha HCR, Garcia WB. Estudo dos flebotomíneos (Diptera, Psychodidae), em área de leishmaniose tegumentar, no Estado de Mato Grosso do Sul, Brasil. Revista de Saúde Pública 30:115-128, 1996.

8. Gomes AC, Galati EAB. Flebotomíneos de Londrina, Paraná (Brasil) e observações ecológicas sobre algumas espécies. Revista de Saúde Pública 11:284-87, 1977.

9. Hueck K. As florestas da América do Sul. Editora da Universidade de Brasília/ Editora Polígono AS, São Paulo, 1972.

10. Instituto Brasileiro de Geografia e Estatística. Atlas do Maranhão. Edição em Convênio com o Governo do Estado do Maranhão, Rio de Janeiro, 1984.

11. Lainson R. Epidemiologia e ecologia da leishmaniose tegumentar na Amazônia. Hiléia Médica, Belém 3:35-40, 1981.

12. Lainson R. Leishmaniasis. In: Steele JH (ed) Handbook séries in zoonoses, p.41-103, 1983.

13. Lainson R, Shaw JJ. The role of animals in the epidemiology of South American leishmaniasis. 
In: Lumsden, Evans DA (eds) Biology of the kinetoplastida, Academic Press, London, p.1-116, 1979.

14. Martins AV, Morales-Farias EN. Sobre a distribuição geográfica dos flebotomíneos americanos (Diptera, Psychodidae, Phlebotominae). Revista Brasileira de Biologia 32:361-371, 1972.

15. Mayrink W, Williams P, Coelho MV, Dias M, Martins AV, Magalhães PA, Costa CA, Falcão AR, Melo MN, Falcão AL. Epidemiology of dermal leishmaniasis in the Rio Doce Valley. State of Minas Gerais, Brazil. American Tropical Medicine and Parasitology 73:123-137, 1979.

16. Nascimento MDSB, Fiori BIP, Carneiro LS, GonçalvesFilho M, Buratini MN. Estado atual da leishmaniose visceral no Maranhão: Aspectos epidemiológicos preliminares. Revista da Sociedade Brasileira de Medicina Tropical 27:242, 1994.

17. Nimer E. Climatologia do Brasil. Instituto Brasileiro de Geografia e Estatística, Rio de Janeiro, 1979.

18. Pereira GFM, Fonseca HHR. Leishmaniose tegumentar americana: epidemiologia e controle. Revista da Sociedade Brasileira de Medicina Tropical 27:45-50, 1994.

19. Ready PD, Day JC, Souza AA, Rangel EF, Davies CR. Mitochondrial DNA characterization of populations of Lutzomyia whitmani (Diptera, Psychodidae) incriminated in the peridomestic and silvatic transmission of Leishmania species in Brazil. Bulletin of Entomological Research 87:187-195, 1997.

20. Ready PD, Lainson R, Shaw JJ. Leishmaniasis in Brazil: $X X$. Prevalence of enzootic rodent leishmaniasis (Leishmania mexicana amazonensis), and apparent absence of "pians bois" (Le. Brasiliensis guyanensis), in plantations of introduced tree species and in other nonclimax forests in eastern Amazônia. Transactions of the Royal Society of Tropical Medicine and Hygiene 77:775785, 1983.
21. Ready PD, Souza AA, Rebêlo JMM, Day JC, Silveira F, Campbell-Lendrum D, Davies CR, Costa JML. Phylogenetic species and domesticity of Lutzomyia whitmaniat the south-east boundary of Amazonian Brazil. Transactions of the Royal Society of Tropical Medicine and Hygiene 92:159-160, 1998.

22. Rebêlo JMM, Mendes WA, Costa JML, Cavaleiro N. Lista preliminar das espécies do gênero Lutzomyia França, 1924 (Psychodidae, Phlebotominae) do Estado do Maranhão, Brasil. Caderno de Saúde Pública 12:545-549, 1996.

23. Ryan L. Flebótomos do Estado do Pará, Brasil. Documento Técnico do Instituto Evandro Chagas, Belém, Pará, Brasil, 1986.

24. Silva AR, Martins G, Melo JEM, Araújo JP, Mendes MG. Surto epidêmico de leishmaniose tegumentar americana na colonização agrícola de Buriticupu (MA). Revista do Instituto de Medicina Tropical 21:45-50, 1979.

25. Souza A, Ishikawa E, Braga R, Silveira F, Lainson R, Shaw J. Psychodopygus complexus, a new vector of Leishmania braziliensis to humans in Pará State, Brazil. Transactions of the Royal Society of Tropical Medicine and Hygiene 90:112-113, 1996.

26. Teodoro U, Salvia Filho V, Lima EM, Spinosa RP, Barbosa AC. Ferreira MEMC, Silveira TGV. Flebotomíneos em área de transmissão de leishmaniose tegumentar na região norte do Estado do Paraná-Brasil: Seasonal variation and nocturnal activity. Revista de Saúde Pública, 27:190-194, 1993.

27. Vexenat JA, Barretto AC, Cuba CC, Marsden P. Características epidemiológicas da leishmaniose tegumentar americana em uma região endêmica do Estado da Bahia. III. Fauna flebotomínica. Memórias do Instituto Oswaldo Cruz 81:293-301, 1986. 